\title{
Modal Limit Cycle Control for Variable Stiffness Actuated Robots
}

\author{
Dominic Lakatos, Gianluca Garofalo, Florian Petit, Christian Ott, and Alin Albu-Schäffer
}

\begin{abstract}
This paper presents a control approach to stabilize limit cycle motions along a mechanical mode of variable stiffness actuated (VSA) robots. Thereby, first a computed torque PD controller with gravity and Coriolis/centrifugal compensation shapes a desired dynamics, which is decoupled in terms of modal coordinates. Then an asymptotically stable limit cycle is generated on the link side dynamics for a selected mode. Finally, the modal control approach first introduced for rigid robots is extended to the VSA case. This is done by a joint torque controller, which decouples the torque dynamics from the link side dynamics. Stability and convergence are proven for the dynamics resulting from each feedback control. Furthermore, the energy efficiency of the proposed approach is verified by simulation and experiments on the VSA robotic arm DLR Hand Arm System.
\end{abstract}

\section{INTRODUCTION}

The emergence of variable stiffness actuators (VSA) in robotic systems offers the capability to perform highly dynamical, explosive or cyclic motions efficiently. Due to serial, tunable elastic elements in the joints, the output power of VSA robots is no longer restricted by the motors. The elastic energy storage can be exploited to maximize peak force and velocities. Thereby, the intrinsic resonance behavior of the elastic robot can be customized to match the desired motion and/or improve the efficiency of actuation. However, the mechanical implementation of VSA usually introduces strong nonlinearities in addition to the rigid robot dynamics. Therefore, the goal is to control such highly dynamical motion while taking these nonlinear dynamics of the plant into account.

The generation of explosive motions using analytical optimal control solutions is addressed in [1]. A method to embody desired (periodic) motions while tuning the stiffness optimally is proposed by [2]. Both approaches are currently feasible for a single joint system. To control dynamical, multi-joint motions, numerical, constrained optimization is applied to generate motor trajectories [3], [4]. The approach is currently limited due to the increasing computational complexity and number of local minima with the number of degrees of freedom.

In our previous work [5] we proposed a method to exploit modal properties of the mechanical system for motion generation. However, the approach was limited to the generation of motor trajectories for serial elastic actuated robots with linear stiffness in the joints. Due to the presence of modal coupling terms and the open-loop structure, this method is limited to quasi-linear systems, motions on the first mode of the system, and tasks where external disturbances are not expected.

Here, we rigorously extend this method for stabilizing modal limit cycles for multi-joint VSA robots. In contrast to the above mentioned approaches, we aim to produce a control for intrinsically periodic tasks, such as hitting, drumming,

The authors are with the Institute of Robotics and Mechatronics, German Aerospace Center (DLR), D-82234 Oberpfaffenhofen, Germany \{dominic.lakatos, gianluca.garofalo, florian.petit, christian.ott, alin.albuschaeffer\}@dlr.de

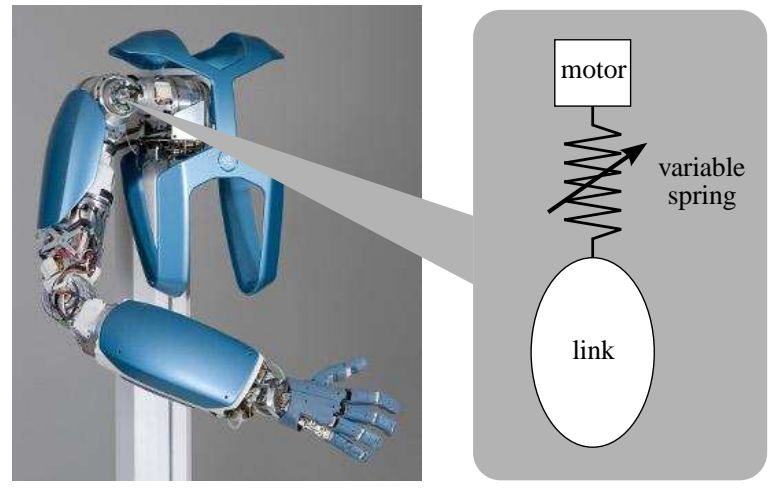

variable stiffness actuator joint torque

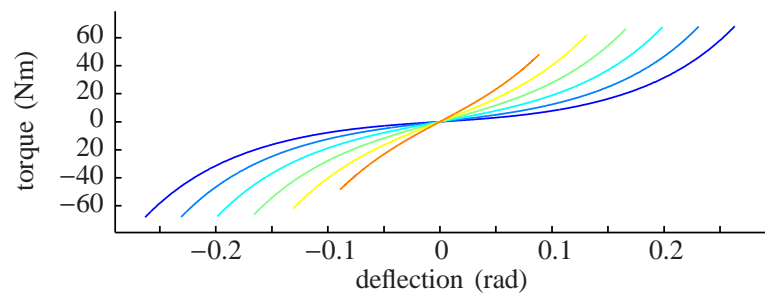

Fig. 1. DLR Hand Arm System and basic VSA principle.

or walking and running [6], where it is more important that the desired orbit is attractive, rather than to track a predefined trajectory accurately [7], [8]. Due to the occurrence of disturbances and impacts the attractive behavior is of major importance in these tasks. The generation of stable limit cycles for rigid robots have been already studied in [9], [10], [11]. While the basic theory on limit cycles is concerned with twodimensional vector fields [12], [13], [14], [15], a main issue is to generalize for motions in the robot's higher-dimensional configuration space. This can be achieved by choosing one position-velocity pair for the limit cycle, while the remaining states have to be constrained, e.g. by virtual constraints based on feedback linearization [10] or null space decomposition [11].

In the present work, the considered class of mechanical systems are VSA robots. Each joint of the robot is composed of a motor and link side mass. Both masses are connected via a nonlinear, tunable spring as depicted in Fig. 1. Due to the elastic energy storages, the plant itself exhibits natural oscillatory dynamics [16]. We identify these so-called oscillation modes and apply a control which decouples these modes and constrains the motion on one of the invariant manifolds. Using these dynamic constraints, we are able to stabilize limit cycles along the natural dynamics of the multi-joint robot. In particular, this leads to an increase in between motor and linkside power.

The paper is organized as follows: In Section II a control 
to first shape and then decouple the dynamics based on eigenmode analysis is proposed. Since the uncontrolled system is nonlinearly coupled, it is accounted for modal cross-coupling terms due to the state dependency of the mass matrix (and stiffness matrix). Furthermore, it is proven that the obtained dynamics are asymptotically stable (even in the absence of Coriolis/centrifugal terms). Then a controller to generate an attractive limit cycle on a particular mode is presented in Section III. Therefore, the total energy corresponding to this mode is regulated to a desired level. Finally, the approach is applied to the VSA case using a feedback controller which decouples the joint torque dynamics from the link side motion (Section IV). Moreover, the accuracy of the modal decoupling is validated in simulations and the performance and efficiency of the whole approach are demonstrated in experiments for a complete VSA robotic arm (Section V). A brief conclusion is given in Section VI.

\section{Modal CONTROLLeR DESIGN}

\section{A. Modal decoupling}

The modal decoupling is introduced for the link side dynamics, given by

$$
\boldsymbol{M}(\boldsymbol{q}) \ddot{\boldsymbol{q}}+\boldsymbol{C}(\boldsymbol{q}, \dot{\boldsymbol{q}}) \dot{\boldsymbol{q}}+\boldsymbol{g}(\boldsymbol{q})=\tau .
$$

Herein $\boldsymbol{q} \in \mathbb{R}^{n}$ are joint positions, $\boldsymbol{M}(\boldsymbol{q}) \in \mathbb{R}^{n \times n}$ is the symmetric and positive definite inertia matrix, $\boldsymbol{C}(\boldsymbol{q}, \dot{\boldsymbol{q}}) \dot{\boldsymbol{q}}$ the Coriolis/centrifugal forces and $\boldsymbol{g}(\boldsymbol{q})$ the gravitational forces.

Before performing the modal decoupling we consider the computed torque plus PD control

$$
\boldsymbol{\tau}=\boldsymbol{u}_{q}+\boldsymbol{C}(\boldsymbol{q}, \dot{\boldsymbol{q}}) \dot{\boldsymbol{q}}+\boldsymbol{g}(\boldsymbol{q})+\boldsymbol{M}(\boldsymbol{q}) \ddot{\boldsymbol{q}}_{d}-\boldsymbol{D}_{d}(\boldsymbol{q}) \dot{\tilde{\boldsymbol{q}}}-\boldsymbol{K}_{d}(\boldsymbol{q}) \tilde{\boldsymbol{q}}
$$

which compensates for Coriolis/centrifugal and gravity effects. The controller is able to track a desired trajectory $\boldsymbol{q}_{d}(t)$ in joint coordinates, where $\tilde{\boldsymbol{q}}=\boldsymbol{q}-\boldsymbol{q}_{d}$ is the tracking error. In addition to the symmetric and positive definite stiffness and damping matrix $\boldsymbol{D}_{d}(\boldsymbol{q})$ and $\boldsymbol{K}_{d}(\boldsymbol{q})$, we introduce an intermediate control input $\boldsymbol{u}_{q}$. Substituting the control (2) in the plant dynamics (1), leads to the desired closed-loop error dynamics

$$
\boldsymbol{M}(\boldsymbol{q}) \ddot{\tilde{\boldsymbol{q}}}+\boldsymbol{D}_{d}(\boldsymbol{q}) \dot{\tilde{\boldsymbol{q}}}+\boldsymbol{K}_{d}(\boldsymbol{q}) \tilde{\boldsymbol{q}}=\boldsymbol{u}_{q},
$$

where the following property holds:

Property 1: For robot manipulators with rotational joints, the controller gain $\boldsymbol{K}_{d}(\boldsymbol{q})$ can be designed such that the generalized eigenvalues $\lambda_{i}\left(\boldsymbol{K}_{d}(\boldsymbol{q}), \boldsymbol{M}(\boldsymbol{q})\right)$ keep bounded, i.e.

$$
\begin{array}{r}
0<\lambda_{\min , K M} \leq \lambda_{i}\left(\boldsymbol{K}_{d}(\boldsymbol{q}), \boldsymbol{M}(\boldsymbol{q})\right) \leq \lambda_{\max , K M}<\infty, \\
\forall i=1, \ldots, n, \forall \boldsymbol{q} \in \mathbb{R}^{n}
\end{array}
$$

where

$$
\begin{aligned}
\lambda_{\min , K M} & :=\inf _{\boldsymbol{q} \in \boldsymbol{Q}} \lambda_{\min }\left(\boldsymbol{K}_{d}(\boldsymbol{q}), \boldsymbol{M}(\boldsymbol{q})\right), \\
\lambda_{\max , K M} & :=\sup _{\boldsymbol{q} \in \boldsymbol{Q}} \lambda_{\max }\left(\boldsymbol{K}_{d}(\boldsymbol{q}), \boldsymbol{M}(\boldsymbol{q})\right) .
\end{aligned}
$$

Now, we derive the decoupling coordinate transformation for the system (3). Therefore, we make use of the generalized eigenvalue problem known from matrix algebra, see [17] and [18]:

Lemma 1: Given a symmetric and positive definite matrix $\boldsymbol{A} \in \mathbb{R}^{n \times n}$ and a symmetric matrix $\boldsymbol{B} \in \mathbb{R}^{n \times n}$. Then there exist a non-singular matrix $\boldsymbol{W} \in \mathbb{R}^{n \times n}$ and a diagonal matrix $\boldsymbol{B}_{W} \in$ $\mathbb{R}^{n \times n}$, such that $\boldsymbol{W}^{-T} \boldsymbol{W}^{-1}=\boldsymbol{A}$ and $\boldsymbol{W}^{-T} \boldsymbol{B}_{W} \boldsymbol{W}^{-1}=\boldsymbol{B}$.

If we now apply this lemma to $\boldsymbol{A} \triangleq \boldsymbol{M}(\boldsymbol{q})$ and $\boldsymbol{B} \triangleq \boldsymbol{K}_{d}(\boldsymbol{q})$, we obtain the transformation

$$
z=\boldsymbol{W}(\boldsymbol{q})^{-1} \tilde{\boldsymbol{q}}
$$

which maps the joint error coordinates $\tilde{\boldsymbol{q}}$ to the modal coordinates $z$. In order to transform the system (3) to modal coordinates, we rewrite (6) as

$$
\tilde{q}=W(q) z
$$

and derive w.r.t. time:

$$
\begin{aligned}
& \dot{\tilde{q}}=\boldsymbol{W}(\boldsymbol{q}) \dot{z}+\dot{\boldsymbol{W}}(\boldsymbol{q}) z, \\
& \ddot{\tilde{\boldsymbol{q}}}=\boldsymbol{W}(\boldsymbol{q}) \ddot{z}+2 \dot{\boldsymbol{W}}(\boldsymbol{q}) \dot{z}+\ddot{\boldsymbol{W}}(\boldsymbol{q}) z .
\end{aligned}
$$

Thereby, $\boldsymbol{W}(\boldsymbol{q})$ depends explicitly on time, i.e. $\boldsymbol{W}\left(\tilde{\boldsymbol{q}}+\boldsymbol{q}_{d}(t)\right):=$ $\boldsymbol{W}(\boldsymbol{q})$.

Remark 1: The transformation matrix $\boldsymbol{W}(\boldsymbol{q})$ is composed of $n$ eigenvectors, which result from the generalized eigenvalue problem $\boldsymbol{K}_{d}(\boldsymbol{q}) \boldsymbol{w}=\lambda \boldsymbol{M}(\boldsymbol{q}) \boldsymbol{w}$. In the case $n>3$ the matrix $\boldsymbol{W}(\boldsymbol{q})$ and consequently the derivative $\dot{\boldsymbol{W}}(\boldsymbol{q})$ can not be computed in closed form. To compute the time derivatives of $\boldsymbol{W}(\boldsymbol{q})$ we use a method, which was proposed by [19] and is described in the Appendix A.

The damping matrix

$$
\boldsymbol{D}_{d}(\boldsymbol{q})=\boldsymbol{W}(\boldsymbol{q})^{-T}\left(2 \operatorname{diag}\left(\xi_{i} \sqrt{\lambda_{i}}\right)\right) \boldsymbol{W}(\boldsymbol{q})^{-1},
$$

is designed in modal coordinates. Herein $\xi_{i} \geq 0$ is a constant, normalized damping factor, referred to as modal damping. By substituting (7)-(9) in (3) we obtain the modal dynamics

$$
\ddot{z}+2 \operatorname{diag}\left(\xi_{i} \sqrt{\lambda_{i}(\boldsymbol{q})}\right) \dot{z}+\operatorname{diag}\left(\lambda_{i}(\boldsymbol{q})\right) z=\boldsymbol{W}(\boldsymbol{q})^{T}\left(\boldsymbol{u}_{q}-\boldsymbol{\gamma}\right)
$$

where

$$
\begin{aligned}
\boldsymbol{I} & =\boldsymbol{W}(\boldsymbol{q})^{T} \boldsymbol{M}(\boldsymbol{q}) \boldsymbol{W}(\boldsymbol{q}) \\
\operatorname{diag}\left(\lambda_{i}(\boldsymbol{q})\right) & =\boldsymbol{W}(\boldsymbol{q})^{T} \boldsymbol{K}_{d}(\boldsymbol{q}) \boldsymbol{W}(\boldsymbol{q}) \\
\boldsymbol{\gamma} & =\left(\boldsymbol{M}(\boldsymbol{q}) \ddot{\boldsymbol{W}}(\boldsymbol{q})+\boldsymbol{D}_{d}(\boldsymbol{q}) \dot{\boldsymbol{W}}(\boldsymbol{q})\right) z+2 \boldsymbol{M}(\boldsymbol{q}) \dot{\boldsymbol{W}}(\boldsymbol{q}) \dot{z}
\end{aligned}
$$

For certain eigenvalue and modal damping distributions and moderate velocities [16], the influence of the remaining coupling terms is weak and can be neglected. In particular, if the rate of change of the eigenvalues is moderate, several modal control approaches [20], [21], [22] successfully run without compensating these coupling terms. However, the effect increases for high velocity motions. Since we are interested in generating and stabilizing highly dynamical motions, these coupling terms are cancelled using the control law:

$$
\boldsymbol{u}_{q}=\boldsymbol{W}(\boldsymbol{q})^{-T} \boldsymbol{u}_{z}+\boldsymbol{\gamma}
$$

where $\boldsymbol{u}_{z}$ is an additional intermediate control input. Substituting the control (15) in (11), we obtain $n$ modal subsystems

$$
\ddot{z}+2 \operatorname{diag}\left(\xi_{i} \sqrt{\lambda_{i}(\boldsymbol{q})}\right) \dot{z}+\operatorname{diag}\left(\lambda_{i}(\boldsymbol{q})\right) z=\boldsymbol{u}_{z},
$$

in terms of the modal coordinates $z$. 


\section{B. Stability analysis}

To proof stability of the homogeneous closed loop dynamics (16) we consider a positive definite Lyapunov function candidate $V_{i}$ for each decoupled subsystem and deduce stability if each time derivative $\dot{V}_{i}$ is negative definite, i.e. $V=\sum_{i} V_{i}>$ $0 \Leftarrow V_{i}>0, \dot{V}=\sum_{i} \dot{V}_{i}<0 \Leftarrow \dot{V}_{i}<0, \forall(z, \dot{z} \neq \mathbf{0})$. Thus, let us consider the $i$-th decoupled subsystem

$$
\ddot{z}_{i}+2 \xi_{i} \sqrt{\lambda_{i}(\boldsymbol{q})} \dot{z}_{i}+\lambda_{i}(\boldsymbol{q}) z_{i}=0
$$

isolated. With the state vector $\boldsymbol{x}_{i}=\left(z_{i}, \dot{z}_{i}\right)^{T}$, a positive definite Lyapunov function candidate is given by

$$
V_{i}\left(\boldsymbol{x}_{i}\right)=\frac{1}{2} \boldsymbol{x}_{i}^{T}\left[\begin{array}{cc}
c_{2} & \frac{c_{1}}{2} \\
\frac{c_{1}}{2} & 1
\end{array}\right] \boldsymbol{x}_{i},
$$

where $c_{1}, c_{2}$ are positive constants and $c_{2}>c_{1}^{2} / 4$. The derivative of the Lyapunov function

$$
\dot{V}_{i}\left(\boldsymbol{x}_{i}, \boldsymbol{q}\right)=-\frac{1}{2} \boldsymbol{x}_{i}^{T} \boldsymbol{H}_{i}\left(\lambda_{i}(\boldsymbol{q})\right) \boldsymbol{x}_{i},
$$

is negative definite, if the matrix

$$
\boldsymbol{H}_{i}\left(\lambda_{i}(\boldsymbol{q})\right)=\left[\begin{array}{cc}
c_{1} \lambda_{i}(\boldsymbol{q}) & \lambda_{i}(\boldsymbol{q})+c_{1} \xi_{i} \sqrt{\lambda_{i}(\boldsymbol{q})}-c_{2} \\
\operatorname{sym} . & 4 \xi_{i} \sqrt{\lambda_{i}(\boldsymbol{q})}-c_{1}
\end{array}\right]
$$

is positive definite. We can conclude that the equilibrium point $\boldsymbol{x}_{i}=0$ of (17) is asymptotically stable, if the leading principal minors of $\boldsymbol{H}_{i}\left(\lambda_{i}(\boldsymbol{q})\right)$ are strictly positive (in $\boldsymbol{q}$ ). That is $\forall \boldsymbol{q} \in Q$

$$
\begin{aligned}
c_{1} \lambda_{i}(\boldsymbol{q}) & >0, \\
\operatorname{det}\left(\boldsymbol{H}_{i}\left(\lambda_{i}(\boldsymbol{q})\right)\right) & >0 .
\end{aligned}
$$

Remark 2: Condition (21) is always fulfilled by definition of $c_{1}$ and Property 1. Condition (22) implies that positive constants $c_{1}$ and $c_{2}$ can be found such that

$$
\forall \lambda_{i}, 0<\lambda_{i, \min , K M} \leq \lambda_{i} \leq \lambda_{i, \max , K M}<\infty, \operatorname{det}\left(\boldsymbol{H}_{i}\left(\lambda_{i}\right)\right)>0
$$

where

$$
\begin{aligned}
\lambda_{i, \min , K M} & :=\inf _{\boldsymbol{q} \in \boldsymbol{Q}} \lambda_{i, \min }\left(\boldsymbol{K}_{d}(\boldsymbol{q}), \boldsymbol{M}(\boldsymbol{q})\right), \\
\lambda_{i, \max , K M} & :=\sup _{\boldsymbol{q} \in \boldsymbol{Q}} \lambda_{i, \max }\left(\boldsymbol{K}_{d}(\boldsymbol{q}), \boldsymbol{M}(\boldsymbol{q})\right),
\end{aligned}
$$

hold. The above conditions are depicted in Fig. 2(a). For certain $\xi_{i}, \lambda_{i, \min , K M}$ and $\lambda_{i, \max , K M}$ the iso-contour curves $\operatorname{det}\left(\boldsymbol{H}_{i}\left(\lambda_{i, \min , K M}\right)\right)=\operatorname{det}\left(\boldsymbol{H}_{i}\left(\lambda_{i, \max , K M}\right)\right)=0$ are plotted w.r.t. $c_{1}$ and $c_{2}$. Thereby, the area within the intersection of both curves determines $c_{1}$ and $c_{2}$ such that condition (22) is fulfilled. Moreover, Fig. 2(b) shows that for $c_{1}$ and $c_{2}$ chosen w.r.t. Fig. 2(a), condition (22) continuously holds within the bounds.

As detailed in the Appendix B, $\xi_{i}$ determines the domain $\mathcal{D}:=] \lambda_{i, 1}, \lambda_{i, 2}$ [ such that for $\lambda_{i}(\boldsymbol{q}) \in \mathcal{D}$ conditions (21) and (22) are fulfilled. Thereby, one can find constants $c_{1}, c_{2}$ that minimize and maximize the lower bound $\lambda_{i, 1}$ and upper bound $\lambda_{i, 2}$, respectively. Then, given the "stable" domain $\mathcal{D}, \boldsymbol{K}_{d}(\boldsymbol{q})$ has to be designed such that $\lambda_{i, \min , K M}>\lambda_{i, 1}$ and $\lambda_{i, \max , K M}<$ $\lambda_{i, 2}$.

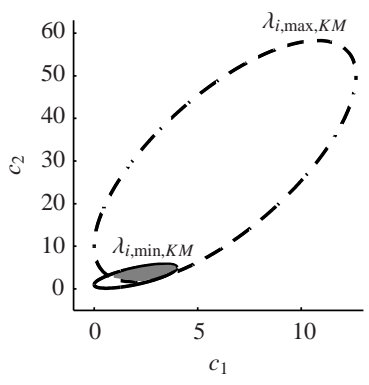

(a)

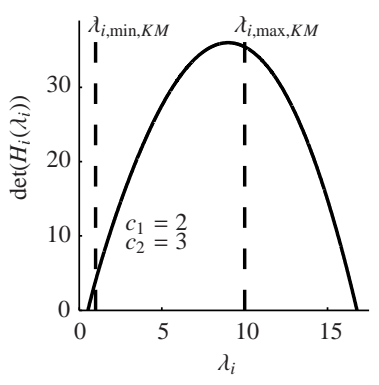

(b)
Fig. 2. Determination of coefficients $c_{1}$ and $c_{2}$.

\section{Comments}

The control law (2) cancels out the Coriolis/centrifugal effects $\boldsymbol{C}(\boldsymbol{q}, \dot{\boldsymbol{q}}) \dot{\boldsymbol{q}}$. This is necessary to accurately decouple the dynamics. From a viewpoint of passivity the skew-symmetric property of $\dot{\boldsymbol{M}}(\boldsymbol{q})-2 \boldsymbol{C}(\boldsymbol{q}, \dot{\boldsymbol{q}})$, commonly considered to prove stability of such type of mechanical systems, is lost. However, a method is proposed to prove stability based on the boundedness of the modal stiffness and damping term. These boundedness conditions do not restrict the domain of attraction, since the eigenvalues $\lambda_{i}\left(\boldsymbol{M}(\boldsymbol{q}), \boldsymbol{K}_{d}(\boldsymbol{q})\right)$ are naturally bounded. Therefore, asymptotic stability in the whole state-space can be proven for a certain choice of the desired stiffness $\boldsymbol{K}_{d}(\boldsymbol{q})$ (without preserving the above mentioned skew-symmetric property). We can summarize that for the origin $(z, \dot{z})=\mathbf{0}$ of the homogeneous dynamics (16), global asymptotic stability can be guaranteed under the conditions (21) and (22).

\section{ENERGY BASED LIMIT CYCLE CONTROL}

Let us assume that we want to produce an asymptotic stable limit cycle for the $k$-th decoupled system in (16). Choosing $\xi_{k}=0$ and dividing by $\lambda_{k}(t)>0 \forall t$, the system becomes

$$
\frac{1}{\lambda_{k}(t)} \ddot{z}_{k}+z_{k}=\frac{1}{\lambda_{k}(t)} u_{z_{k}},
$$

with state $\left(z_{k}, \dot{z}_{k}\right) \in \mathbb{R}^{2}$. Similarly to what was done in [11] let us consider the scalar function

$$
H\left(t, z_{k}, \dot{z}_{k}\right)=\frac{1}{2 \lambda_{k}(t)} \dot{z}_{k}^{2}+\frac{1}{2} z_{k}^{2},
$$

with time derivative along the trajectories of (23):

$$
\dot{H}\left(t, z_{k}, \dot{z}_{k}\right)=\frac{1}{\lambda_{k}(t)} u_{z_{k}} \dot{z}_{k}-\frac{\dot{\lambda}_{k}(t)}{2 \lambda_{k}(t)^{2}} \dot{z}_{k}^{2},
$$

and choose the input as

$$
u_{z_{k}}=\lambda_{k}(t)\left(-k_{V} \tilde{H}\left(t, z_{k}, \dot{z}_{k}\right) \dot{z}_{k}+\frac{\dot{\lambda}_{k}(t)}{2 \lambda_{k}(t)^{2}} \dot{z}_{k}\right),
$$

where $k_{V}>0, \tilde{H}\left(t, z_{k}, \dot{z}_{k}\right)=H\left(t, z_{k}, \dot{z}_{k}\right)-H_{d}$ and $H_{d}>0$, such that the derivative of $H\left(t, z_{k}, \dot{z}_{k}\right)$ results in

$$
\dot{H}\left(t, z_{k}, \dot{z}_{k}\right)=-k_{V} \tilde{H}\left(t, z_{k}, \dot{z}_{k}\right) \dot{z}_{k}^{2} .
$$

The system (23), unlike the one considered in [11], is non-autonomous and consequently we cannot apply LaSalle theorem. Nevertheless with a similar argumentation we can 
prove that it has an asymptotically stable limit cycle $\Omega=$ $\left\{z_{k}, \dot{z}_{k} \mid H\left(t, z_{k}, \dot{z}_{k}\right)=H_{d}\right\}$. We have to show stability and attractiveness.

Uniform stability: Choosing as Lyapunov function the continuously differentiable function

$$
V\left(t, z_{k}, \dot{z}_{k}\right)=\frac{1}{2} \tilde{H}\left(t, z_{k}, \dot{z}_{k}\right)^{2},
$$

with:

- $V(t, \Omega)=0$

- $S_{1}\left(z_{k}, \dot{z}_{k}\right) \leq V\left(t, z_{k}, \dot{z}_{k}\right) \leq S_{2}\left(z_{k}, \dot{z}_{k}\right)$

- $\dot{V}\left(t, z_{k}, \dot{z}_{k}\right)=-k_{V} \tilde{H}\left(t, z_{k}, \dot{z}_{k}\right)^{2} \dot{z}_{k}^{2} \leq 0$

$\forall t \geq 0$ and $\forall\left(z_{k}, \dot{z}_{k}\right) \in \mathbb{R}^{2}$, where

$$
\begin{aligned}
& S_{1}\left(z_{k}, \dot{z}_{k}\right)=\frac{1}{2}\left(\frac{1}{2 \lambda_{k, \text { max }}} \dot{z}_{k}^{2}+\frac{1}{2} z_{k}^{2}-H_{d}\right)^{2}, \\
& S_{2}\left(z_{k}, \dot{z}_{k}\right)=\frac{1}{2}\left(\frac{1}{2 \lambda_{k, \text { min }}} \dot{z}_{k}^{2}+\frac{1}{2} z_{k}^{2}-H_{d}\right)^{2},
\end{aligned}
$$

are positive definite functions on $\mathbb{R}^{2}$, we conclude that $\Omega$ is uniformly stable.

Attractiveness: To prove attractiveness we will use Barbalat's lemma (see, e.g. [23]). We have already shown that $V\left(t, z_{k}, \dot{z}_{k}\right)$ is bounded from below and that $\dot{V}\left(t, z_{k}, \dot{z}_{k}\right)$ is negative semidefinite. What is missing in order to apply Barbalat's lemma is that $\ddot{V}\left(t, z_{k}, \dot{z}_{k}\right)$ is bounded. This follows from $\ddot{V}\left(t, z_{k}, \dot{z}_{k}\right)=-2 k_{V} \tilde{H}\left(t, z_{k}, \dot{z}_{k}\right)^{2}\left(k_{V} \dot{z}_{k}+\dot{z}_{k} \ddot{z}_{k}\right)$, which is bounded, since (23) is stable. So we conclude that

$$
\lim _{t \rightarrow \infty} \dot{V}\left(t, z_{k}, \dot{z}_{k}\right)=0 \text {. }
$$

Let $B_{\epsilon}(\Omega)$ be a neighbourhood of $\Omega$, such that

$$
\left(z_{k}=0, \dot{z}_{k}=0\right) \notin B_{\epsilon}(\Omega) .
$$

Since $\Omega$ is stable, we can choose the initial condition such that the solution is always in $B_{\epsilon}(\Omega)$. Moreover we have shown that either $\dot{z}_{k} \rightarrow 0$ or $H\left(t, z_{k}, \dot{z}_{k}\right) \rightarrow H_{d}$ as $t \rightarrow \infty$, but since the system cannot converge to $\left(z_{k} \neq 0, \dot{z}_{k}=0\right)$ and $\left(z_{k}=0, \dot{z}_{k}=0\right) \notin B_{\epsilon}(\Omega)$ then we conclude that the solution converges to $\Omega=\left\{z_{k}, \dot{z}_{k} \mid H\left(t, z_{k}, \dot{z}_{k}\right)=H_{d}\right\}$.

Remark 3: The controller (26) is composed of a term to regulate the energy along the $k$-th mode and an additional, Coriolis/centrifugal like term $\boldsymbol{C}_{\text {mod }}$ to compensate for the $k$ th diagonal entry of the derivative of the modal mass matrix $\dot{\boldsymbol{\Lambda}}=$ $\boldsymbol{C}_{\text {mod }}+\boldsymbol{C}_{\text {mod }}^{T}$, where $\boldsymbol{\Lambda}=\operatorname{diag}\left(1 / \lambda_{i}(t)\right)$. However, in (2) the Coriolis/centrifugal vector $\boldsymbol{C}(\boldsymbol{q}, \dot{\boldsymbol{q}})$ was already cancelled and here this term is introduced again for the $k$-th mode. This is as the modal analysis requires $\boldsymbol{C}(\boldsymbol{q}, \dot{\boldsymbol{q}})$ to be cancelled but it is necessary for the passivity of the controller (26).

\section{EXTENSION TO THE VARIABLE STIFFNESS ACTUATED CASE}

In the previous sections the modal decoupling and limit cycle controller were introduced for the rigid robot case, where it was assumed that we can directly access the joint torques. In this section we will extend the methods for a VSA robot arm, where only the motor torque is accessible. In the rigid robot case the oscillatory dynamics were achieved by a virtual elastic potential using control. Instead, the VSA robot consist of a variable spring between the motor and the link. Thus, the plant itself exhibits oscillatory dynamics. In particular, the stiffness of the springs can be tuned to match with the oscillatory dynamics shaped by control. Thereby, the performance and efficiency will increase.

\section{A. Dynamic modeling of VSA robots}

VSA robots are under actuated Euler-Lagrange systems and can be generally described by dynamic equations of the form [24], [25]:

$$
\boldsymbol{M}(\boldsymbol{x}) \ddot{\boldsymbol{x}}+\boldsymbol{c}(\boldsymbol{x}, \dot{\boldsymbol{x}})+\frac{\partial U(\boldsymbol{x})}{\partial \boldsymbol{x}}=\boldsymbol{Q} .
$$

Herein $\boldsymbol{x} \in \mathbb{R}^{n}$ are generalized coordinates and $\boldsymbol{Q} \in \mathbb{R}^{n}$ is a non-conservative, generalized force dual to $\dot{x}$. The potential energy

$$
U(x)=U_{g}(\boldsymbol{x})+U_{\psi}(\boldsymbol{x}),
$$

is composed of the gravity potential $U_{g}(\boldsymbol{x})$ and the spring potential $U_{\psi}(\boldsymbol{x})$. Furthermore $\boldsymbol{M}(\boldsymbol{x}) \in \mathbb{R}^{n \times n}$ is the symmetric and positive definite inertia matrix and $\boldsymbol{c}(\boldsymbol{x}, \dot{\boldsymbol{x}}) \in \mathbb{R}^{n}$ a vector of Coriolis/centrifugal forces of the complete robot, respectively. The design of VSA robots involves that not all of the system states are directly actuated. Therefore, let us partition the states $\boldsymbol{x}=\left(\boldsymbol{\theta}^{T}, \boldsymbol{q}^{T}\right)^{T}$ as $\boldsymbol{\theta} \in \mathbb{R}^{k}$ being directly actuated states (referred to as motor positions) and $\boldsymbol{q} \in \mathbb{R}^{n-k}$ being indirectly actuated states (referred to as link positions). Then the generalized force

$$
\boldsymbol{Q}=\left[\begin{array}{c}
\boldsymbol{\tau}_{m} \\
\boldsymbol{\tau}_{\text {ext }}
\end{array}\right]
$$

consists of the control input $\tau_{m} \in \mathbb{R}^{k}$ and the externally applied force $\tau_{\text {ext }}$. Before deriving a controller for the joint torque dynamics, the general dynamic model (30) is customized based on simplifying assumptions fully justified for the DLR Hand Arm System [26] and briefly summarized as following:

- the coupling inertias in between motor and link side can be neglected ${ }^{1}$.

- The electrical motor dynamics are sufficiently fast to be neglected ${ }^{2}$

- Stiffness adjustments are performed quasi statically. Accordingly, the simplified motor dynamics have the form:

$$
\begin{aligned}
\boldsymbol{B} \ddot{\boldsymbol{\theta}}_{q}+\boldsymbol{\tau} & =\boldsymbol{\tau}_{m}, \\
\boldsymbol{\tau} & =\frac{\partial U_{\psi}\left(\boldsymbol{\theta}_{q}, \boldsymbol{q}, \boldsymbol{\theta}_{\psi}\right)}{\partial \boldsymbol{\theta}_{q}}=\boldsymbol{\psi}\left(\boldsymbol{\theta}_{q}-\boldsymbol{q}, \boldsymbol{\theta}_{\psi}\right) .
\end{aligned}
$$

Herein $\boldsymbol{B}$ is a constant, diagonal, and positive definite matrix of motor inertias, $\boldsymbol{\theta}_{q} \in \mathbb{R}^{k}$ are motor coordinates, and $\boldsymbol{\theta}_{\psi} \in \mathbb{R}^{k}$ are constant parameters for the stiffness adjustment. Using the above mentioned assumptions, the link side dynamics remains as introduced in (1).

\section{B. Decoupled torque control}

Now, let us derive a controller to track a desired joint torque. The basic concept of the controller is to decouple the joint torque dynamics from the dynamics of the joint position, i.e. to obtain a system in triangular form. The approach was proposed in [30] for the case of constant joint stiffness and will be extended to the VSA case as follows.

\footnotetext{
${ }^{1}$ This assumption is fulfilled in the presence of high gear ratios, cf. [27].

${ }^{2}$ Singular perturbation assumption, cf. e.g. [28], [29].
} 
To relate the system (1), (33), and (34) we first rewrite (33) in terms of joint torques $\tau$. Therefore, the strictly monotone and odd spring functions (34) are inverted, i.e.

$$
\boldsymbol{\theta}_{q}-\boldsymbol{q}=\boldsymbol{\psi}(\boldsymbol{\tau})^{-1}
$$

Then deriving the inverse spring function twice w.r.t. time and substituting the result in (33), one obtains

$$
\boldsymbol{B}\left(\frac{\partial \boldsymbol{\psi}(\boldsymbol{\tau})^{-1}}{\partial \boldsymbol{\tau}} \ddot{\boldsymbol{\tau}}+\frac{\mathrm{d}}{\mathrm{d} t}\left(\frac{\partial \psi(\tau)^{-1}}{\partial \tau}\right) \dot{\tau}\right)+\boldsymbol{\tau}=\tau_{m}-\boldsymbol{B} \ddot{\boldsymbol{q}}
$$

In the above dynamics the term $\boldsymbol{B} \ddot{\boldsymbol{q}}$ remains. In order to cancel out this term, the control law

$$
\boldsymbol{\tau}_{m}=\boldsymbol{u}_{\tau}+\boldsymbol{B} \ddot{\boldsymbol{q}},
$$

can be considered ${ }^{3}$. Herein $\boldsymbol{u}_{\tau}$ is an intermediate control input for the resulting decoupled torque dynamics:

$$
\boldsymbol{B} \frac{\partial \boldsymbol{\psi}(\tau)^{-1}}{\partial \tau} \ddot{\tau}+\boldsymbol{B} \frac{\mathrm{d}}{\mathrm{d} t}\left(\frac{\partial \psi(\tau)^{-1}}{\partial \tau}\right) \dot{\tau}+\boldsymbol{\tau}=\boldsymbol{u}_{\tau}
$$

Finally, the torque error $\boldsymbol{e}_{\tau}=\boldsymbol{\tau}-\boldsymbol{\tau}_{d}$ can be introduced. Then the control law

$$
\begin{aligned}
\boldsymbol{u}_{\tau}= & \boldsymbol{\tau}_{d}+\boldsymbol{B} \frac{\mathrm{d}}{\mathrm{d} t}\left(\frac{\partial \boldsymbol{\psi}(\boldsymbol{\tau})^{-1}}{\partial \boldsymbol{\tau}}\right) \dot{\boldsymbol{\tau}} \\
& +\boldsymbol{B} \frac{\partial \boldsymbol{\psi}(\boldsymbol{\tau})^{-1}}{\partial \boldsymbol{\tau}}\left(\ddot{\boldsymbol{\tau}}_{d}-\boldsymbol{D}_{\tau} \dot{\boldsymbol{e}}_{\tau}-\boldsymbol{K}_{\tau} \boldsymbol{e}_{\tau}\right)
\end{aligned}
$$

leads to the error dynamics

$$
\ddot{\boldsymbol{e}}_{\tau}+\boldsymbol{D}_{\tau} \dot{\boldsymbol{e}}_{\tau}+\left[\boldsymbol{K}_{\tau}+\left(\frac{\partial \psi(\tau)^{-1}}{\partial \tau}\right)^{-1} \boldsymbol{B}^{-1}\right] \boldsymbol{e}_{\tau}=\mathbf{0},
$$

where $\boldsymbol{K}_{\tau}$ and $\boldsymbol{D}_{\tau}$ are diagonal, positive definite gain matrices, respectively. Substituting $\boldsymbol{\tau}=\boldsymbol{\tau}_{d}+\boldsymbol{e}_{\tau}$ in the link side dynamics (1), one obtains

$$
\boldsymbol{M}(\boldsymbol{q}) \ddot{\boldsymbol{q}}+\boldsymbol{C}(\boldsymbol{q}, \dot{\boldsymbol{q}}) \dot{\boldsymbol{q}}+\boldsymbol{g}(\boldsymbol{q})=\tau_{d}+\boldsymbol{e}_{\tau}
$$

From (40) and (41) it can be seen that the desired torque equals the actual torque if the torque error converges to zero. The desired dynamics derived in the last sections can be achieved by replacing $\tau$ by $\tau_{d}$ in (2).

Remark 4: In the case of the DLR Hand Arm System, the spring functions (34) and consequently the inverted spring functions (35) consist of no couplings in between the joints, i.e. $\psi_{i}^{-1}=\psi_{i}\left(\tau_{i}\right)^{-1}$. Thus, the torque error dynamics itself are decoupled. In order to prove asymptotic stability for the $(n-k)$ decoupled systems (40) we can consider the $i$ th scalar system isolated. Notice that the term $\left(\partial \psi_{i}\left(\tau_{i}\right)^{-1} / \partial \tau_{i}\right)^{-1} \equiv$ $\partial \psi_{i}\left(\theta_{i}-q_{i}\right) / \partial \theta_{i} \equiv \partial^{2} U_{\psi}(\theta, \boldsymbol{q}) / \partial \theta_{i}^{2}$, is the second derivative of the spring potential and bounded from above and below, i.e. $0<c_{3} \leq\left(\partial \psi_{i}\left(\tau_{i}\right)^{-1} / \partial \tau_{i}\right)^{-1} \leq c_{4}<\infty$. Thus, we can consider a similar Lyapunov function as already used in Section II-B, to prove asymptotic stability of the torque error dynamics (40).

\footnotetext{
${ }^{3}$ The controller (37) requires the second time derivative of the measured link position, which can be obtained from (1), since the internal torques $\tau$ are measurable.
}

\section{EXPERIMENTAL VALIDATION AND PERFORMANCE ANALYSIS}

In this section we first show the influence of the remaining modal coupling terms by simulations. Then, we experimentally apply the presented approach on a VSA robotic arm. Finally, we demonstrate performance and efficiency.

\section{A. Simulation}

The relevance of the modal decoupling controller (15) proposed in Section II is demonstrated for a double pendulum like robotic arm in simulation. Therefore, it is assumed that control (2) and (15) can be directly commanded to the rigid body dynamics (1). The following parameters are considered. Both links of the robot are equal: link length $l_{1 / 2}=0.4 \mathrm{~m}$ and point masses $m_{1 / 2}=5.0 \mathrm{~kg}$ placed at half the link length. The desired stiffness is $\boldsymbol{K}_{d}=\operatorname{diag}(20,10) \mathrm{Nm} / \mathrm{rad}$ and the modal damping $\boldsymbol{\xi}=(0.7,0)$. The responses to a steplike excitation $\hat{\boldsymbol{u}}_{z}=(100,0)^{T}$ in terms of normalized modal forces are simulated in Matlab/Simulink ${ }^{\circledR}$ using the fixedstep solver ode1 (Euler forward integration) with a fixed stepsize of $0.0005 \mathrm{~s}$. Notice that only the mode that is excited is damped. Modal positions and velocities for two cases are shown in Fig. 3. In the first case depicted in Fig. (3)(a)-(b) the modal coupling terms $\gamma$ (cf. (14)) are compensated. It can be clearly seen that the modal motion is accurately decoupled. In the second case Fig. (3)(c)-(d) the modal coupling terms are maintained in the system (i.e. $\gamma \equiv \mathbf{0}$ in the decoupling controller (15)). It can be seen that both modes are excited. Since the step-like excitation acts only on the first mode, this is due to the modal coupling term $\gamma$.

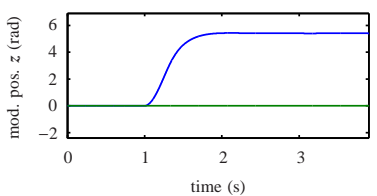

(a)

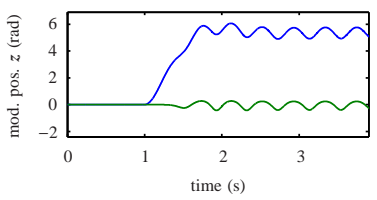

(c)

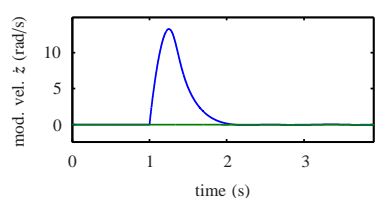

(b)

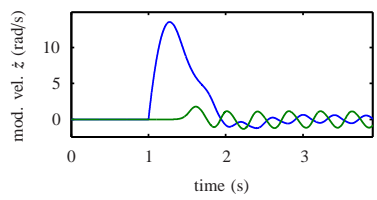

(d)
Fig. 3. Influence of the modal coupling term $\gamma$. The first / second row displays modal positions and velocities, where $\gamma$ is / is not canceled out.

\section{B. Experiments}

The complete approach (i.e. modal decoupling, limit cycle, and torque feedback control) was tested for the first four VSA joints of the DLR Hand Arm System ${ }^{4}$. For all experiments the desired stiffness was $\boldsymbol{K}_{d}=\operatorname{diag}(150,150,150,150) \mathrm{Nm} / \mathrm{rad}$ and the modal damping $\xi_{k}=0$ for the $k$-th excited mode and $\xi_{i}=1$ for the remaining modes. The parameters of the torque tracking controller were $\boldsymbol{K}_{\tau}=\operatorname{diag}(10,5,5,5) 1 / \mathrm{s}^{2}$ and $\boldsymbol{D}_{\tau}=\operatorname{diag}\left(2 \xi_{\tau} \omega_{\tau, i}\right) 1 / \mathrm{s}$, where $\xi_{\tau}=1$ and $\omega_{\tau}=\left(\boldsymbol{K}_{\tau}+\right.$

\footnotetext{
${ }^{4} \mathrm{~A}$ video attachment is provided, showing the experimental tests
} 
$\left.\left(\partial \boldsymbol{\psi}(\tau)^{-1} / \partial \tau\right)^{-1} \boldsymbol{B}^{-1}\right)^{1 / 2}$. To overcome the numerical computation of higher derivatives for measured signals, only the desired joint torque $\tau_{d}(t)$ and the first derivative $\dot{\tau}_{d}(t)$ had been provided to the torque tracking controller. The limit cycle controller was applied either to the first or second mode. Thereby the desired total energy was $H_{d}=0.08$ or $H_{d}=0.035$, respectively, and the limit cycle feedback gain $k_{V}=1.5$. In order to induce the limit cycle motion the robotic arm had been manually pushed from one of the initial configurations: $\boldsymbol{q}_{d}=(-\pi / 6,0,0, \pi / 3)$ for the first and $\boldsymbol{q}_{d}=(\pi / 6, \pi / 6, \pi / 12, \pi / 3)$ for the second mode.

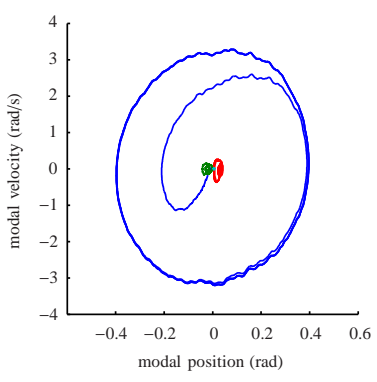

(a) First mode motion

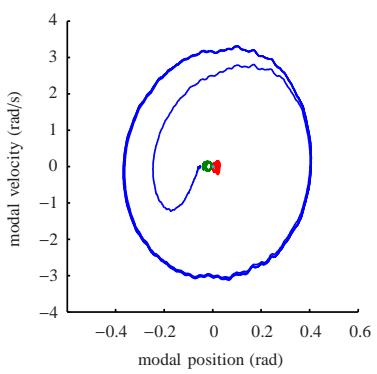

(c) First mode motion

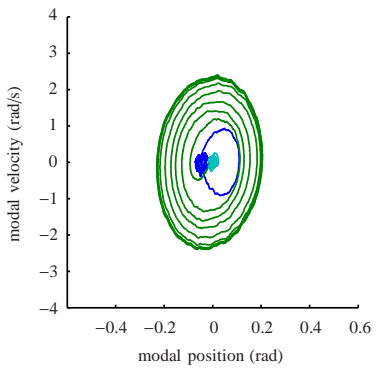

(e) Second mode motion

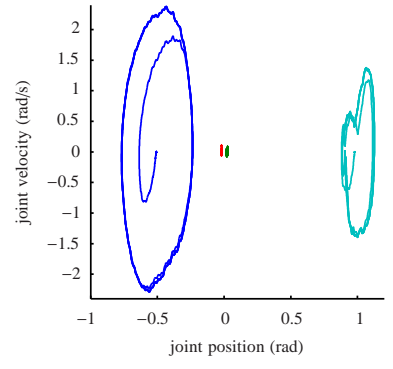

(b) Joint motion

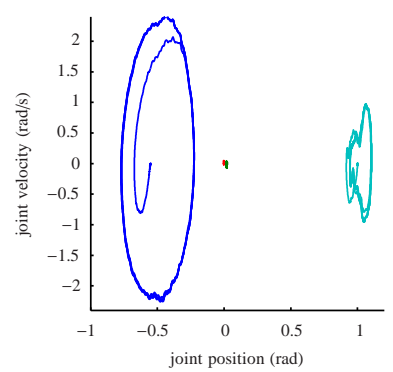

(d) Joint motion

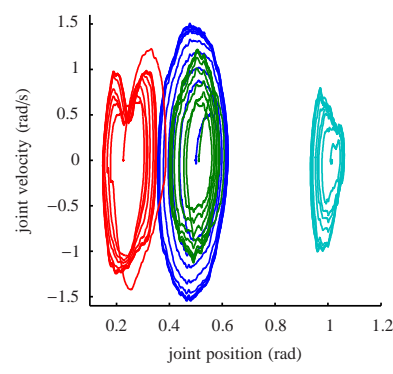

(f) Joint motion
Fig. 4. Experimental validation of the complete approach for a VSA robotic arm (first four joints of the DLR Hand Arm System). On the left and right side phase plots of modal and joint motions are depicted, respectively.

The experimentally recorded first mode motions for two different stiffness presets (i.e. low stiffness $\boldsymbol{\theta}_{\psi}=\boldsymbol{\theta}_{\psi, \min }$ and high stiffness $\left.\boldsymbol{\theta}_{\psi}=0.5 \boldsymbol{\theta}_{\psi, \max }\right)$ are shown in Fig. 4(a)-(d). For both stiffness presets the modal motion is similar. This is as the desired dynamics is equal. In both cases, the motion of the first mode asymptotically approaches the limit cycle, while the motion of the remaining modes stays within a small region around the origin of the modal state space. The motion in terms of link positions involves mainly the first and

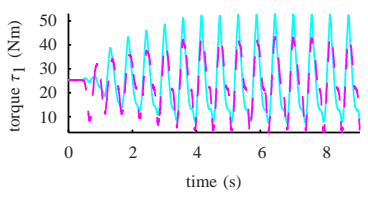

(a)

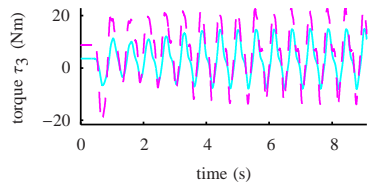

(c) (b)

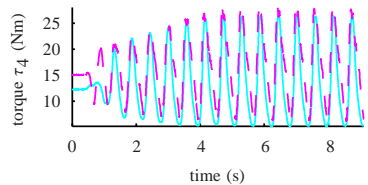

(d)

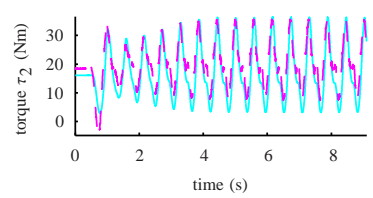

Fig. 5. Tracking performance of the torque controller. The desired (dashed lines) and actual (solid lines) torques are displayed for each joint. The data corresponds to motions depicted in Fig. 4(e)-(f).

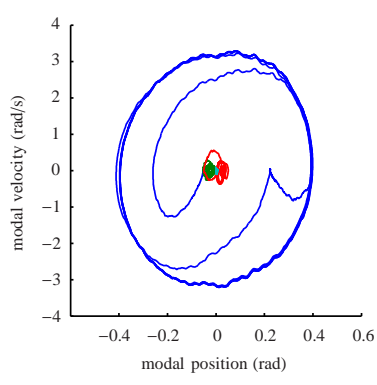

(a) Modal motion

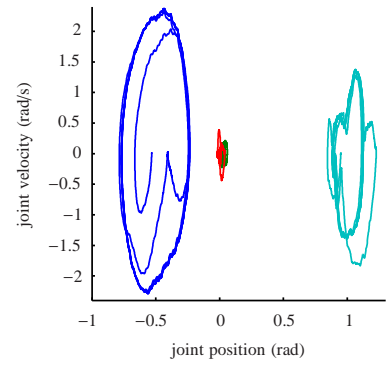

(b) Joint motion
Fig. 6. Attractive behavior of the controller in the presence of external disturbances. The VSA robotic arm was manually stopped and released during motion.

fourth joint. Moreover, second mode motions are shown in Fig. 4(e)-(f). This mode involves the motion of all joints. The corresponding tracking performance of the torque controller is shown in Fig. 5. During the regulation phase (where the robot maintains in the initial configuration), a constant tracking error can be observed. This can be due to friction (not considered for the torque controller). In order to reduce the tracking error during the high acceleration phases of the desired torque, one should consider also the second time derivative of the desired torque. Furthermore, the attractive behavior of the modal limit cycle controller is depicted in Fig. 6. Thereby, the robotic arm was externally disturbed by catching and releasing the wrist manually during motion. The motion converges back to the limit cycle in less than a half cycle.

\section{Performance measures}

In the introduction of this paper we stated that VSA robots are able to execute power efficient motions. In particular, the efficiency can be increased by adjusting the VSA stiffness such that it matches the desired dynamics. To verify the efficiency of the proposed approach, we introduce a performance measure based on power considerations. Let us therefore consider the 
total power of the VSA robot dynamics (1) and (33)-(34):

$$
P_{\text {tot }}=\underbrace{\dot{\boldsymbol{q}}^{T} \boldsymbol{\tau}_{\text {tot }}}_{P_{\text {link }}}+\underbrace{\dot{\boldsymbol{\theta}}^{T} \boldsymbol{\tau}_{m}}_{P_{\text {motor }}}+\underbrace{\dot{\boldsymbol{q}}^{T} \frac{\partial U_{\psi}(\boldsymbol{\theta}, \boldsymbol{q})}{\partial \boldsymbol{q}}}_{P_{\text {stf }}}+P_{\mathrm{dis}} .
$$

Herein, $P_{\text {link }}$ (where $\tau_{\text {tot }}=\tau+\tau_{\text {ext }}$ ) and $P_{\text {motor }}$ is the power of the links and motors, respectively. $P_{\text {stf }}$ is the power transmitted via the springs and $P_{\text {dis }}$ the dissipated power. The motion generated using the proposed approach is periodic and therefore also the input- and output power will be periodic. Additionally, in practice the power of the motors is not limited due to peaks with short duration. In order to account for this, we define the performance measure as the ratio of root-mean-square (RMS) like values of the input and output power:

$$
\alpha=\left(\sqrt{\int_{0}^{t}\left(P_{\text {link }}(\tau)\right)^{2} \mathrm{~d} \tau}\right) /\left(\sqrt{\int_{0}^{t}\left(P_{\text {motor }}(\tau)\right)^{2} \mathrm{~d} \tau}\right) .
$$

For the same modal limit cycle dynamics, the performance in the case of low and high VSA stiffness presets is compared. Therefore, $\alpha_{\text {low }}$ and $\alpha_{\text {high }}$ was evaluated for the experimental recordings corresponding to motions shown in Fig. 4(a)-(b) and Fig. 4(c)-(d), respectively. The time evolutions of $\alpha_{\text {low }}$ and $\alpha_{\text {high }}$ plotted in Fig. 7 are similar for both cases and explained as follows. During the resting phase the motor and link power is zero and consequently the performance measures are meaningless. As the robot is externally disturbed $\alpha \gg 1$ due to the external power input. Then the controller is active and the value undercuts $\alpha=1$ before approaching stationary values of $\alpha_{\text {low }} \approx 1.8$ and $\alpha_{\text {high }} \approx 2.3$. In the stationary phase the effective motor power is less than the link power. The difference is provided by the elastic elements. Simply speaking, the motor performs less motion than the link as shown in Fig. 8. In particular, customizing the VSA stiffness for the desired dynamics leads to increasing efficiency. Therefore, we can conclude power efficiency for the embodied limit cycle motion.

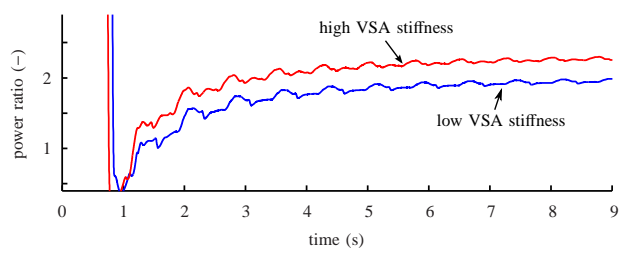

Fig. 7. Performance measures evaluated for limit cycle motions corresponding to Fig. 4(a)-(d). The ratio of RMS values of link and motor power for two different VSA adjustments are plotted over time.

\section{Conclusion}

This paper presents an approach to generate and control modal limit cycles for variable stiffness actuated robots. Therefore, a link side controller is designed, which enables accurate modal decoupling and attraction to a limit cycle. A torque controller ensures tracking of the desired dynamics. Stability and convergence are proven.

The approach is practically verified in simulation with a rigid-body double pendulum and in experiments with the VSA

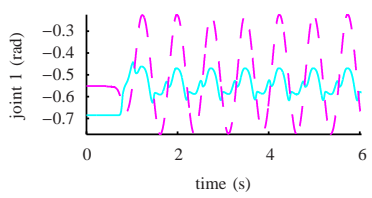

(a)

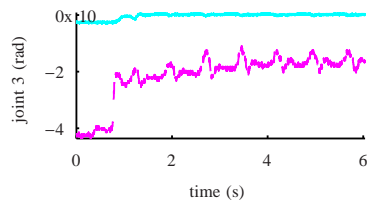

(c)

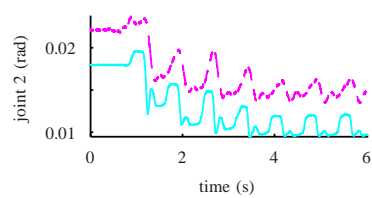

(b)

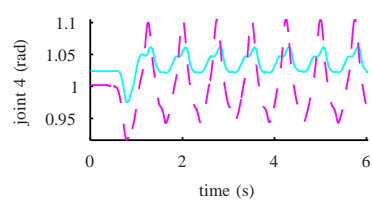

(d)
Fig. 8. Motor position $\theta_{i}$ (solid line) and link side position $q_{i}$ (dashed line) for the $i$ th joint. The data corresponds to motions depicted in Fig. 4(c)-(d).

robotic arm DLR Hand Arm System. It is demonstrated that the approach accurately separates the modal dynamics. Even if the system is externally disturbed the motion converges back to the modal limit cycle. Furthermore, it is experimentally validated that the natural dynamics of the plant can be varied (by statically adjusting the joint stiffness) to closely match the desired modal dynamics. Thereby, even the power efficiency is increased.

The approach can be applied to cyclic motion tasks, where performance, efficiency, and immunity to external disturbances are more important than to accurately track a predefined motion. For example a pick and place task is mainly defined by two pairs of position and velocity. In between these points the motion has only to be fast. How to customize the mode shape to a predefined task is part of our future work.

\section{ACKNOWLEDGMENT}

This work has been partially funded by the European Commission's Seventh Framework Programme as part of the project SAPHARI (Grant no. 287513). The second and the fourth author acknowledge support by the Initiative and Networking Fund of the Helmholtz Association through a Helmholtz Young Investigators Group (Grant no. VH-NG808).

\section{ApPendix A}

\section{DERIVATIVES OF GENERALIZED EIGENVECTORS}

Given two symmetric matrices $\boldsymbol{K}, \boldsymbol{M} \in \mathbb{R}^{n \times n}$ with $\boldsymbol{M}$ positive definite. Consider the following generalized eigenvalue problem

$$
\begin{aligned}
\boldsymbol{K} \boldsymbol{w} & =\lambda \boldsymbol{M} \boldsymbol{w}, \\
\boldsymbol{w}^{T} \boldsymbol{M} \boldsymbol{w} & =1,
\end{aligned}
$$

where (45) defines a normalization for the eigenvectors. Assume that all the quantities in (44) are functions of time. The goal is to compute the time derivative of the couple $\left(\lambda_{k} ; \boldsymbol{w}_{k}\right)$, which is a solution of (44)-(45) with multiplicity one. Derive both sides of (44) and rearrange as

$$
\left(\boldsymbol{K}-\lambda_{k} \boldsymbol{M}\right) \dot{\boldsymbol{w}}_{k}=\dot{\lambda}_{k} \boldsymbol{M} \boldsymbol{w}_{k}-\left(\dot{\boldsymbol{K}}-\lambda_{k} \dot{\boldsymbol{M}}\right) \boldsymbol{w}_{k} .
$$


Pre-multiplying (46) by $\boldsymbol{w}_{k}^{T}$ and taking into account that $\boldsymbol{w}_{k}^{T}\left(\boldsymbol{K}-\lambda_{k} \boldsymbol{M}\right)=\mathbf{0}$ and $\boldsymbol{w}_{k}^{T} \boldsymbol{M} \boldsymbol{w}_{k}=1$, leads to the derivative of $\lambda_{k}$ as

$$
\dot{\lambda}_{k}=\boldsymbol{w}_{k}^{T}\left(\dot{\boldsymbol{K}}-\lambda_{k} \dot{\boldsymbol{M}}\right) \boldsymbol{w}_{k}
$$

To compute $\dot{\boldsymbol{w}}_{k},(47)$ is subsituted in (46):

$$
\left(\boldsymbol{K}-\lambda_{k} \boldsymbol{M}\right) \dot{\boldsymbol{w}}_{k}=\left(\boldsymbol{M} \boldsymbol{w}_{k} \boldsymbol{w}_{k}^{T}-\boldsymbol{I}\right)\left(\dot{\boldsymbol{K}}-\lambda_{k} \dot{\boldsymbol{M}}\right) \boldsymbol{w}_{k},
$$

and then one of the equation in (48) is replaced by the derivative of (45):

$$
2 \boldsymbol{w}_{k}^{T} \boldsymbol{M} \dot{\boldsymbol{w}}_{k}=-\boldsymbol{w}_{k}^{T} \dot{\boldsymbol{M}} \boldsymbol{w}_{k}
$$

The last step is necessary because of $\left(\boldsymbol{K}-\lambda_{k} \boldsymbol{M}\right)$ is singular.

\section{APPENDIX B}

\section{Proof OF THE EXISTENCE OF A "STABLE" DOMAIN FOR $\lambda_{i}$}

We prove that there exists a domain $\mathcal{D}:=] \lambda_{i, 1}, \lambda_{i, 2}[$ such that for $\lambda_{i}(\boldsymbol{q}) \in \mathcal{D}$, condition (22) is fulfilled.

Proof: Rewrite condition (22) in terms of $\omega:=\sqrt{\lambda_{i}(\boldsymbol{q})}$. That is $p(\omega)>0$, where

$$
p(\omega)=-\omega^{4}+2 c_{1} \xi \omega^{3}-\left[\left(1+\xi^{2}\right) c_{1}-2 c_{2}\right] \omega^{2}+2 \xi c_{1} c_{2} \omega-c_{2}^{2}
$$

and for convenience of notation $\xi:=\xi_{i}$. Since the leading coefficient of $p(\omega)$ is negative,

$$
\lim _{\omega \rightarrow-\infty} p(\omega)=\lim _{\omega \rightarrow+\infty} p(\omega)=-\infty .
$$

Moreover, the solution of $p(\omega)=0$ can be expressed as

$$
\begin{aligned}
& \omega_{1,2}=\frac{1}{2} \xi c_{1}+\sqrt{\alpha} \pm \frac{1}{2} \sqrt{\left[\left(\xi^{2}-1\right)+4 \sqrt{\alpha} \xi\right] c_{1}}, \\
& \omega_{3,4}=\frac{1}{2} \xi c_{1}-\sqrt{\alpha} \pm \frac{1}{2} \sqrt{\left[\left(\xi^{2}-1\right)-4 \sqrt{\alpha} \xi\right] c_{1}},
\end{aligned}
$$

where $\alpha:=c_{2}-c_{1}^{2} / 4>0$. From (51) it follows that $\omega_{1,2}$ are real if $\left(\xi^{2}-1\right) / \xi \geq-4 \sqrt{\alpha} / c_{1}$. Therefore, $\forall \xi>0, \exists c_{1}, \alpha>0$ such that $\omega_{1,2}$ are real. From (52) it follows that $\omega_{3,4}$ are real if $\left(\xi^{2}-1\right) / \xi \geq 4 \sqrt{\alpha} / c_{1}$. The latter condition shows $\forall \xi, 0<\xi \leq 1$ and $\forall c_{1}, \alpha>0, \omega_{3,4}$ are complex. Moreover, from (51) and (52) it can be seen that $\exists c_{1}, \alpha>0$ such that $\omega_{1,2}$ are real and $\omega_{3,4}$ are complex. Then, $\forall \xi>0, \exists c_{1}, \alpha>0$ such that $p(\omega)$ has exactly two real zeros. Additionally, (50) holds. We can conclude that $\exists \mathcal{D}=] \omega_{1}^{2}, \omega_{2}^{2}\left[\right.$ such that $\forall \lambda_{i}(\boldsymbol{q}) \in \mathcal{D}$, condition (22) is fulfilled.

\section{REFERENCES}

[1] Garabini, Passaglia, Belo, Salaris, and Bicchi, "Optimality principles in variable stiffness control: The vsa hammer." in Proc. IEEE/RSJ Int. Conf. on Intelligent Robots and Systems. IEEE, 2011, pp. 3770-3775.

[2] Visser, Stramigioli, and Bicchi, "Embodying desired behavior in variable stiffness actuators," in Proc. of the 18th IFAC World Congress, 2011.

[3] Braun, Howard, and Vijayakumar, "Exploiting variable stiffness in explosive movement tasks," in Robotics: Science and Systems, 2011.
[4] Haddadin, Huber, and Albu-Schäffer, "Optimal control for exploiting the natural dynamics of variable stiffness robots," in Proc. IEEE Int. Conf. on Robotics and Automation, 2012.

[5] Petit, Lakatos, Friedl, and Albu-Schäffer, "Dynamic trajectory generation for serial elastic actuated robots," in 10th International IFAC Symposium on Robot Control, 2012.

[6] Geyer, Seyfarth, and Blickhan, "Compliant leg behavior explains basic dynamics of walking and running," Proceedings of the Royal Society B, vol. 273 , p. 28612867 , Nov. 2006.

[7] Westervelt, Grizzle, Chevallereau, Choi, and Morris, Feedback Control of Dynamic Bipedal Robot Locomotion. CRC Press, 2007.

[8] Garofalo, Ott, and Albu-Schäffer, "Walking control of fully actuated robots based on the bipedal slip model," in Proc. IEEE Int. Conf. on Robotics and Automation, 2012, pp. 1999-2004.

[9] Duindam and Stramigioli, "Passive asymptotic curve tracking," in Proceedings of the IFAC Workshop on Lagrangian and Hamiltonian Methods for Nonlinear Control, 2003, pp. 229 - 234.

[10] Canudas-de-Wit, Espiau, and Urrea, "Orbital stabilization of underactuated mechanical systems," in Proc. of the 15th IFAC World Congress, 2002.

[11] Garofalo, Ott, and Albu-Schäffer, "Orbital stabilization of mechanical systems through semidefinite Lyapunov functions," accepted for publication at the 2013 American Control Conference.

[12] Nayfeh and Mook, Nonlinear Oscillations. John Wiley \& Sons, 1979.

[13] Guckenheimer and Holmes, Nonlinear Oscillations, Dynamical Systems, and Bifurcations of Vector Fields. Springer-Verlag, 1983.

[14] Strogatz, Nonlinear Dynamics and Chaos. Perseus Books, 1994.

[15] Jordan and Smith, Nonlinear Ordinary Differential Equations: An Introduction for Scientists and Engineers. Oxford University Press, 2007.

[16] Lakatos, Petit, and Albu-Schäffer, "Nonlinear oscillations for cyclic movements in variable impedance actuated robotic arms," accepted for publication at the IEEE Int. Conf. on Robotics and Automation 2013.

[17] Hoffman and Kunze, Linear Algebra. Prentice-Hall, 1971.

[18] Ott, Cartesian Impedance Control of Redundant and Flexible-Joint Robots, Siciliano and Khatib, Eds. Springer, 2008.

[19] Magnus and Neudecker, Matrix Differential Calculus. John Wiley \& Sons, 2007.

[20] Schutter, Torfs, Bruyninckx, and Dutre, "Invariant hybrid force/position control of a velocity controlled robot with compliant end effector using modal decoupling," The International Journal of Robotics Research, vol. 16, pp. 340-356, 1997.

[21] Tien, Albu-Schäffer, and Hirzinger, "Mimo state feedback controller for a flexible joint robot with strong joint coupling," in Proc. IEEE Int. Conf. on Robotics and Automation, 2007.

[22] Petit and Albu-Schäffer, "State feedback damping control for a multi dof variable stiffness robot arm," in Proc. IEEE Int. Conf. on Robotic and Automation, 2011.

[23] Slotine and Li, Applied Nonlinear Control. Prentice Hall, 1991.

[24] Albu-Schäffer, Wolf, Eiberger, Haddadin, Petit, and Chalon, "Dynamic modelling and control of variable stiffness actuators," in Proc. IEEE Int. Conf. on Robotics and Automation, 2010.

[25] Albu-Schäffer, Ott, and Petit, "Constructive energie shaping control for a class of euler-lagrange systems," in 10th International IFAC Symposium on Robot Control, 2012.

[26] Grebenstein et al., "The DLR Hand Arm System," in Proc. IEEE Int. Conf. on Robotics and Automation, 2011.

[27] Spong, "Modeling and control of elastic joint robots," Transactions of the ASME: Journal of Dynamic Systems, Measurement, and Control, vol. 109 , pp. 310-319, 1987.

[28] Kokotovic, Khalil, and O'Reilly, Singular Perturbation Methods in Control: Analysis and Design. Academic Press, London, 1986.

[29] Khalil, Nonlinear Systems, 3rd edn. Prentice Hall, Englewood Cliffs, 2002.

[30] Ott, Albu-Schäffer, Kugi, and Hirzinger, "Decoupling based cartesian impedance control of flexible joint robots," in Proc. IEEE Int. Conf. on Robotics and Automation, 2003. 